\title{
CONSERVATION DU SPERME DE VERRAT APRÈS DILUTION ET MAINTIEN DE SON POUVOIR FECONDANT
}

\author{
PAR
}

\section{F. DU MESNIL DU BUISSON}

Station de recherches de Physiologie animale, C. N. R. Z., Jouy-en-Josas.

\section{INTROD UCTION}

Le développement de l'insémination artificielle porcine ne peut manquer de se produire tant la fécondation d'une truie représente une sujétion pour l'éleveur. Mais le succès de sa mise en pratique est conditionné par deux ordres de connaissances : la physiologie sexuelle de la Truie d'une part, et la conservation " in vitro " du sperme de Verrat après dilution d'autre part.

Nous avons déjà montré que l'important volume de semence éjaculé par le mâle dans l'utérus n'était pas sans conséquence, puisque dans l'utérus dilaté se produit une véritable digestion du sperme. En inséminant avec un volume comparable à celui émis par le mâle, nous avons pu obtenir des succès appréciables dans un essai portant sur 145 Truies (DU Mesnil, du Buisson et Dauzier, I955). Les expériences poursuivies par plusieurs de nos collègues sur la conservation du sperme de Bélier et la dissociation rapide entre les signes extérieurs de la vitalité du sperme et le maintien du pouvoir fécondant (Dauzier, Thibauli et WinteinBERGER, I954, THIBAULT, I955) et des essais sur le sperme de Verrat, qui paraissaient mettre en évidence des caractéristiques spéciales, en particulier quant à la température optimum de conservation, nous ont amenés pendant ces trois dernières années, à poursuivre des recherches systématiques sur la dilution du sperme de cette espèce. En effet, en face de l'énorme littérature scientifique qu'a suscitée l'insémination bovine, on ne trouve que quelques références ayant trait soit à la conservation de la motilité ou du pouvoir fécondant du sperme pur (MIlovanov, I932 ; Mc Kenzie, Miller et Bauguess, I938 ; Ito, Niwa et Kudo, I949; Niwa, Mizuho et Ishikawa, I952 ; Nozaki, Kawase et Niwa, I952), la dilution n'intervenant qu'au moment de l'insémination, soit à 1'ritilisation du jaune d'œeuf pour la conversation du sperme de Verrat (LASley et Philim's, I939 ; Noll, I949 ; Roy, I955 ; Polge, I956).

Les résultats que nous avons accumulés non seulement sur la conser- 
vation de la motilité ou la vitalité des spermatozoïdes, mais aussi sur le maintien de leur pouvoir fécondant après dilution, nous autorisent à publier cette mise au point qui peut faciliter les progrès ultérieurs de 1 'insémination porcine.

\section{MATÉRIEL ET MÉTHODES}

Nous avons pratiqué la collecte sur i I Verrats de I à 4 ans, en utilisant un mannequin et un vagin artificiel muni d'une poire, selon la méthode de Milovanov (I933) et de Rodolfo (I934); dans certains cas, nous avons fractionné l'éjaculat et employé seulement la partie la plus riche en spermatozoïdes. Sauf mention contraire, nous nous sommes placés pour la dilution et la conservation dans les conditions suivantes :

- dilution amenant la concentration en spermatozoïdes à $50000-$ 75000 spermatozoïdes $/ \mathrm{mm}^{3}$, opérée à $32^{\circ} \mathrm{C} \pm \mathrm{r}^{\circ} \mathrm{C}$;

- refroidissement progressif en 3 heures $\mathrm{I} / 2$;

- température de conservation 7 à $8^{\circ} \mathrm{C}$.

Pour l'examen du sperme et spécialement du sperme non dilué, plusieurs auteurs recommandent de réchauffer les échantillons en les agitant à l'air pendant 2 heures à $37^{\circ} \mathrm{C}$ (ITO, Niwa et Kudo, Noll, POLGE). Cela ne nous a pas paru nécessaire pour examiner le sperme frais après la collecte et le sperme conservé dans les milieux à base de jaune d'œuf ou de lait ; nous avons pris comme critère de survie et de motilité, l'aspect microscopique après un réchauffement très rapide (40 secon łes à I minute, sur platine chauffante) entre lame et lamelles.

La motilité a été notée de o à 5 . Chaque observation porte sur 6 collectes dont les spermatozoïdes ont été conservés de manière identique.

\section{RÉSULTATS ET DISCUSSION}

\section{I. - Le dilueur :}

Io Dilueurs salins et dilueurs biologiques. - Notre méthode d'examen ne nous a pas permis de prendre le sperme non dilué comme témoin au cours de la conservation, car dans les conditions d'examen où nous nous sommes placés, la motilité et la survie apparente des spermatozoïdes dans leur milieu d'origine est très faible dès le lendemain de la collecte. De même, la conservation du sperme de Verrat dans des solutions salines équilibrées s'est avérée d'emblée si décevante, que nous n'avons étudié que des dilueurs contenant également des liquides biologiques.

En utilisant le jaune d'œuf dans la proportion de I volume pour 3 volumes de solution, nous avons comparé (fig. I), la valeur des tampons suivants :

- le tampon citrate (SAlisbury, Fuller et Willet, r94I),

- le tampon phosphate (PHILLIPS et LARDy, I940), 
- le tampon bicarbonate (Kampschmid' et Mayer, Herman et DICKERSON, I95I).

Nous avons également comparé à celle des dilueurs précédents, la valeur d'un milieu sans sets, ne contenant que du jaune d'œuf et du glycocolle, selon une formule préconisée par Roy (I955), pour le sperme de Verrat (voir tableau I, tampons A, B, C et II).

\section{TABLEAU I}

\section{Dilueurs}

\begin{tabular}{|c|c|c|c|c|}
\hline \multirow{3}{*}{ Dilueurs } & \multicolumn{4}{|c|}{ Proportion pour roo ce de dilueur } \\
\hline & \multicolumn{2}{|l|}{ Solution aqueuse } & \multicolumn{2}{|c|}{ Milieu Biologique } \\
\hline & $\begin{array}{l}\text { Composition en g pour soo cc } \\
\text { de solution aqueuse }\end{array}$ & Quantité & Désignation & Quan tité \\
\hline $\begin{array}{l}\mathrm{A} \\
\mathrm{B}\end{array}$ & $\begin{array}{l}\text { Citrate de Sodium }: 3 \mathrm{~g} \ldots \ldots \ldots \ldots \ldots \ldots \\
\text { Phosphate monopotassique, o, g et phosphate }\end{array}$ & $75 \mathrm{cc}$ & Jaune d'oeuf & $25 \mathrm{cc}$ \\
\hline & disodique, $\left(\mathrm{r} 2 \mathrm{H}^{2} \mathrm{O}\right), 2 \mathrm{~g} . \ldots \ldots \ldots \ldots \ldots$ & $75 \mathrm{cc}$ & Jaune d'oeuf & $25 \mathrm{cc}$ \\
\hline $\mathrm{C}$ & Bicarbonate de Sodium, 0,2 $\mathrm{g}$ et Glucose $4 \mathrm{~g}$. & $75 \mathrm{cc}$ & Jaune d'oeuf & $25 \mathrm{cc}$ \\
\hline I & Bicarbonate de Sodium, $0,2 \mathrm{~g}$ et Glucose $4 \mathrm{~g}$. & $75 \mathrm{cc}$ & Lait écrémé & $25 \mathrm{cc}$ \\
\hline II & Glycoco $[\mathrm{le}, 3 \mathrm{~g} \ldots \ldots \ldots \ldots \ldots \ldots \ldots \ldots$ & $65 \mathrm{cc}$ & Jaune d'oeuf & $35 \mathrm{cc}$ \\
\hline III & 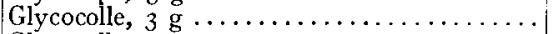 & 50 $\mathrm{cc}$ & Lait écrémé & $50 \mathrm{cc}$ \\
\hline IV & 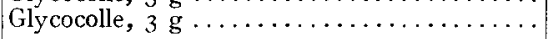 & $75 \mathrm{cc}$ & Lait écrémé & $25 \mathrm{cc}$ \\
\hline
\end{tabular}
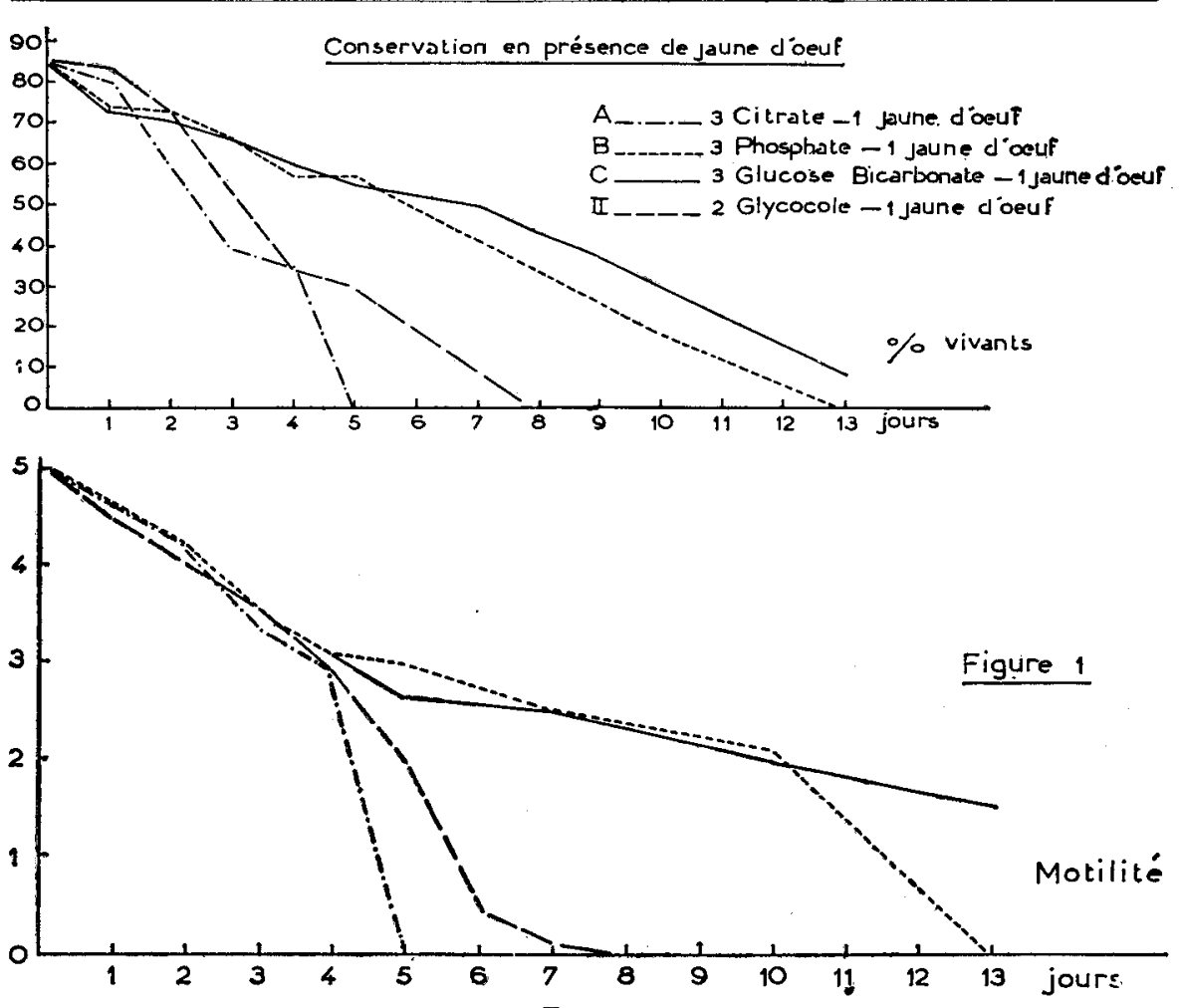

FIG. I. 
Le citrate se montre dès le $2^{\mathrm{e}}$ jour, nettement inférieur aux trois autres milieux et au cours du $4^{\mathrm{e}}$ jour de conservation, la motilité du sperme décroît très rapidement ; le $5^{\mathrm{e}}$ jour, le sperme est apparemment mort.

Les dilueurs phosphate et bicarbonate-glucose permettent une conservation à peu près identique : durant les 2 premiers jours de conservation, le sperme a une très bonne motilité et un pourcentage de vivants supérieur à $70 \mathrm{p}$. Ioo $;$ le $5^{\mathrm{e}}$ jour, on a encore en moyenne $55 \mathrm{p}$. Ioo de vivants dont la motilité est notée de 2,5 à 3 .

Bien que la conservation ne puisse être prolongée au-delà de 8 jours, dans le dilueur au glycocolle (au lieu de 13 pour les 2 précédents) les qualités du sperme pendant les premières 48 heures sont comparables à celles de la semence conservée dans les dilueurs phosphate et bicarbonateglucose.

En résumé, pendant les 2 premiers jours, la motilité et la survie satisfaisantes des spermatozoïdes laissent penser que l'insémination peut être tentée, principalement avec les 3 derniers dilueurs, pour une conservation prolongée, le bicarbonate-glucose et le phosphate se montrent toujours supérieurs au glycocolle et au citrate.

En se plaçant dans des conditions légèrement différentes pour la température, la dilution et la proportion de jaune d'œuf, POLGE examinant, après agitation, ces solutions, arrive à la même conclusion que nous, le citrate lui paraissant le moins bon. Par contre, il considère le glycocolle comme supérieur au phosphate. Il n'a pas utilisé le bicarbonate-glucose.

Pour des raisons d'ordre pratique et économique, en tenant compte des bons résultats obtenus dans l'insémination bovine avec le lait, il était intéressant de comparer la conservation en présence de ce milieu à celle que l'on obtient avec le jaune d'œuf et les meilleurs tampons.

Le lait, préparé à partir de poudre de lait écrémé (Io g/Ioo cc), porté à ébullition Io minutes, a été employé en mélange avec 2 des solutions utilisées précédemment : bicarbonate-glucose et glycocolle, d'abord dans la proportion de I volume de lait pour 3 de solution saline.

Avec le dilueur 3 bicarbonate-glucose-I lait, on obtient durant les 3 premiers jours de conservation (fig. 2 ), une conservation équivalente à celle du sperme dilué dans le 3 bicarbonate-glucose-I jaune d'œuf. Ensuite, le dilueur au lait se montre assez inégal : dans certains échantillons, le sperme est très mauvais dès le $4^{\mathrm{e}}$ jour (40 p. roo-2), dans d'autres, au contraire, la courbe de conservation en présence de lait suit celle du jaune d'œuf, tout en restant sensiblement au-dessous.

L'emploi simultané du glycocolle et du lait (3 glycocolle-I lait), permet la confection d'un dilueur dans lequel le spermatozoïde conserve 
ses qualités durant 24 heures (fig. 2). Il décline ensuite plus rapidement que dans le dilueur 3 bicarbonate-glucose-r lait. Le $3^{\mathrm{e}}$ jour, par exemple, il n'y a plus que 35 à 40 p. Ioo de vivants. Ainsi, l'un et l'autre des dilueurs au lait se montrent inférieurs après quelques heures, à leurs homologues, dans lesquels le lait est remplacé par le jaune d'œuf.
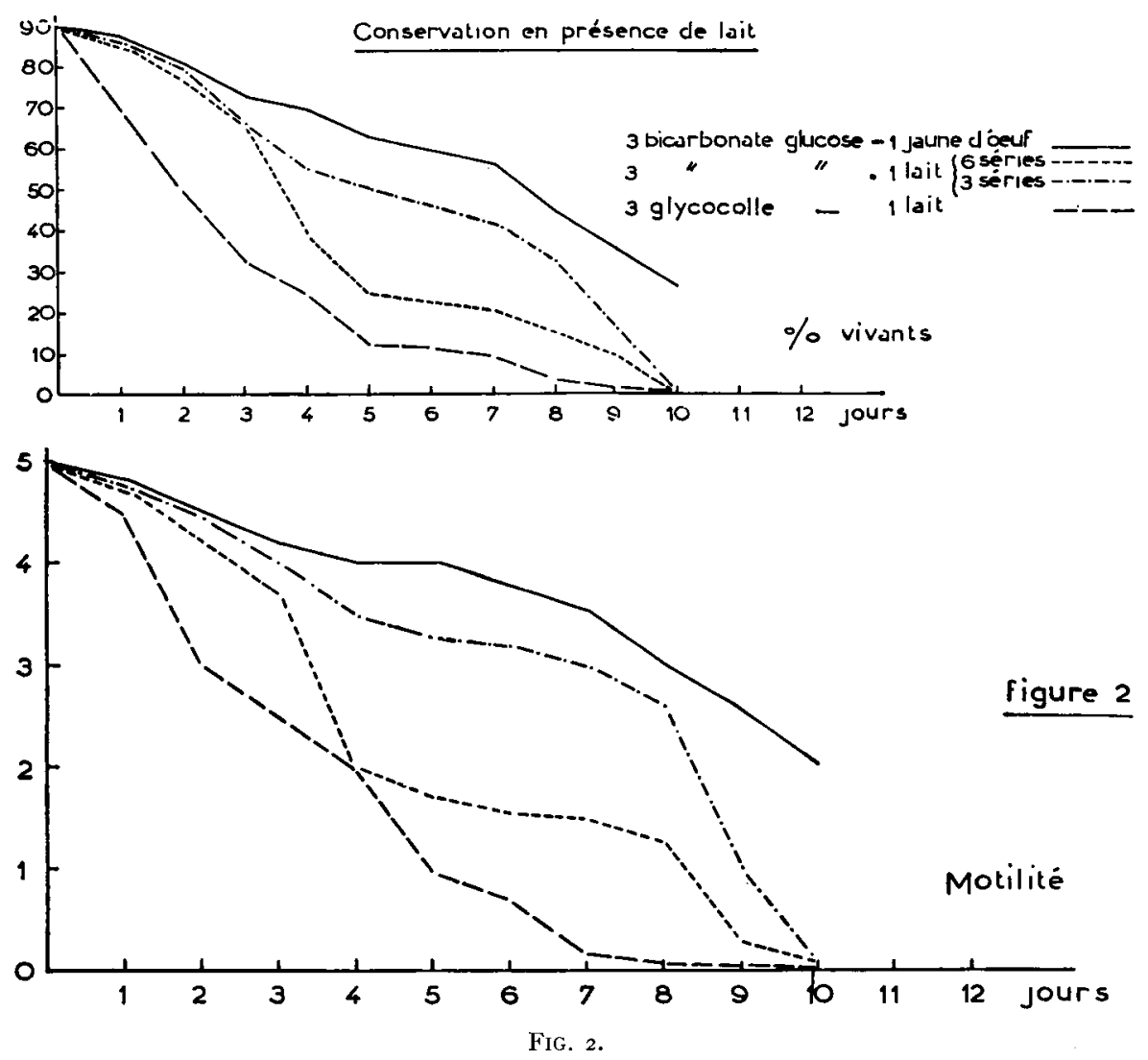

La proportion de lait ou de jaune d'œuf dans les dilueurs peut être modifiée. En le faisant, nous n'avons pas obtenu d'amélioration de conservation du sperme par rapport au témoin ( 25 p. Ioo de milieu biologique, 75 p. Ioo de tampon bicarbonate-glucose). Ainsi, en augmentant dans le dilueur les proportions du milieu biologique $(50 \mathrm{p}$. Ioo de lait ou de jaune d'œuf), on ne note pas de résultats supérieurs au témoin. Au contraire, la limite inférieure que l'on peut atteindre pour la quantité de lait et de jaune d'œuf, n'est pas la même. Avec 5 p. Ioo seulement de jaune d'œuf, on obtient une conservation similaire au témoin (et même légèrement supérieure à partir du $7^{\mathrm{e}}$ jour), alors qu'avec ro p. Ioo de lait on constate un léger fléchissement durant les 3 premiers jours. 
$2^{\circ}$ Emploi de substances existant dans le sperme de Verrat. - A) Esters de la choline: I'action très nettement bénéfique du jaune d'œuf sur la conservation du sperme de Verrat et la mise en évidence dans ce sperme, à la suite des travaux de KAHN et LEVY (1936), de plusieurs estérases (SEKINE, I95I) capables de scinder les composés choliniques, avaient attiré notre attention sur le rôle de la choline et de ses dérivés.

M. Diament (I954) a démontré sur des échantillons de sperme dilué,
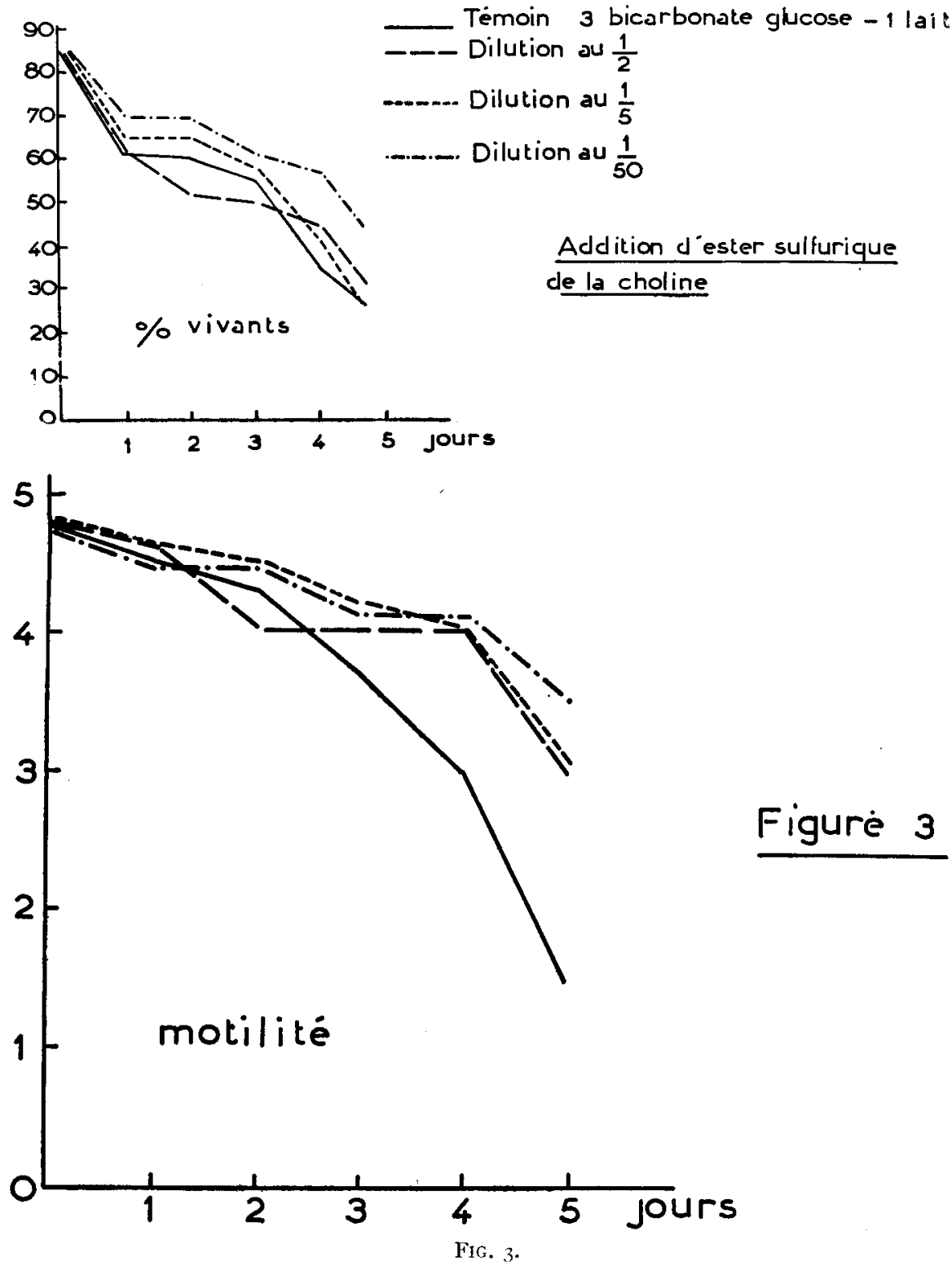
préparés par nous, qu'en présence de bicarbonate, la choline hydrosoluble totale du sperme, aussi bien que celle de l'œuf contenu dans le dilueur, est entièrement retrouvée sous forme de choline libre, après quelques heures d'incubation.

L'existence de ce mécanisme nous a amenés à étudier l'action de plusieurs composés choliniques sur la motilité et la survie des spermatozoïdes " in vivo ".

Le milieu de référence est ici le dilueur 3 bicarbonate-glucose-I lait. A ce dilueur de base, on ajoute des solutions isotoniques de composés choliniques dans la proportion de $\mathrm{I} / 2, \mathrm{I} / 5, \mathrm{I} / 5 \mathrm{O}$.

L'ester phosphorique et glycérophosphorique de la choline de même que la bétaïne neutralisée, sont toxiques pour le sperme aux deux plus fortes concentrations. $\mathrm{Au} \mathrm{I} / 50^{\circ}$, elles sont sans effet.

Le chlorure de choline est nuisible au I/2, sans action au $\mathrm{I} / 5$. Au $\mathrm{I} / 5 \mathrm{O}$, il permet une augmentation de 5 à Io $\mathrm{p}$. Ioo du nombre des vivants, durant les 4 premiers jours de conservation.

I'ester sulfurique de la choline a un effet marqué sur la motilité du sperme. Aux trois doses précitées (fig. 3), il amortit la chute de motilité que l'on enregistre dans le témoin, dès le $4^{\mathrm{e}}$ jour. De plus, le pourcentage de vivants est augmenté durant toute la conservation dans le dilueur au I/5 et surtout au I/5O ; 1'adjonction d'un I/Ioo au dilueur est sans effet. La solution au $x / 2$, bien qu'elle provoque un léger abaissement du pourcentage des vivants au cours de la $2^{\mathrm{e}}$ et de $1 \mathrm{a} 3^{\mathrm{e}}$ journée de conservation, a ensuite également un effet bénéfique.

En résumé, dans les conditions de conservation où nous nous sommes placés, le composé glycéro-phosphoré de la choline, seule forme qui existe dans le sperme de Verrat frais, ne favorise pas la conservation du sperme dilué, quand on l'ajoute au milieu.

Toujours dans ces conditions, l'ester sulfurique a un effet bénéfique; il est possible qu'une diastase existant dans le sperme de Verrat soit capable de scinder l'ester sulfurique de la choline présent dans le dilueur et de permettre au spermatozoïde d'utiliser ce composé cholinique particulier.

B) Inositol: A côté de la choline qui existe certes dans le sperme d'autres Mammifères, mais qu'on trouve sous une forme "spécifique " dans le sperme du Verrat, d'autres composés organiques très rares chez les Mammifères, sont présents dans les sécrétions accessoires du Verratnotamment dans celle des vésicules séminales. Ce sont l'ergothionéine et l'inositol, mis en évidence par MaNN (I95I).

Nous n'avons pas employé l'ergothionéine pour la conservation bien que son rôle de protecteur des liaisons SH (MANN et LEONE, I953), puisse faire envisager son utilisation.

L'inositol (fig. 4), est trop peu soluble pour assurer à lui seul l'isotonie d'un milieu de conservation. En solution additionnée de glucose, 
avec ou sans jaune d'œuf, il ne permet pas une conservation prolongée (courbe $I_{\text {, et }} \mathrm{M}$ ). Au contraire, 1orsqu'on 1'utilise en présence de bicarbonate et de jaune d'œuf, l'inositol assure au sperme une conservation comparable à celle obtenue avec le glucose.
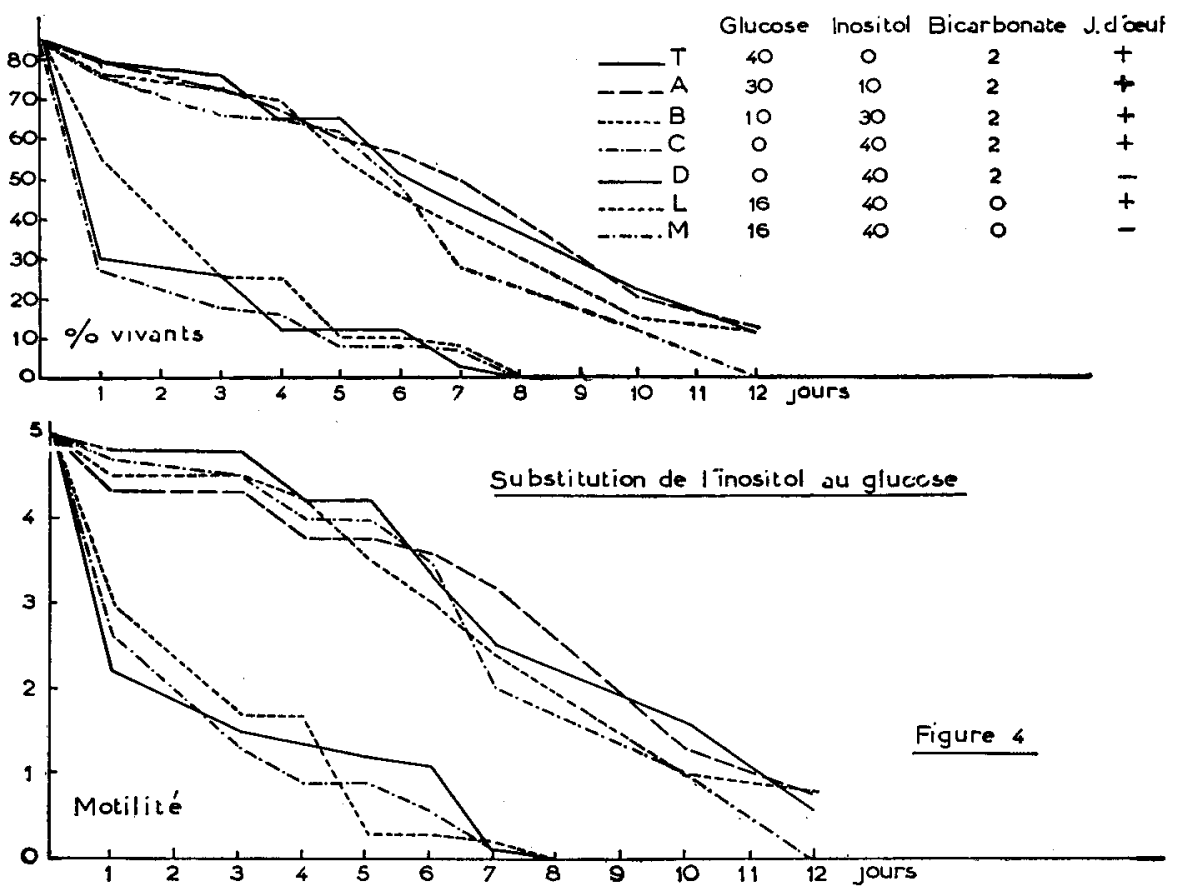

FIG. 4.

Les conditions de conservation sont donc les mêmes dans le 3 bicarbonate-glucose-I jaune d'œuf et dans le 3 bicarbonate-inositol-I jaune d'œuf (inositol substitué à poids égal au glucose).

Sans jaune d'œuf, on observe comme avec la plupart des milieux salins, une forte agglutination et un phénomène de choc dès la dilution (courbe D).

$3^{\circ}$ Emploi des antibiotiques. - Un autre aspect de la composition du dilueur reste à examiner : c'est l'adjonction possible de substances à activité bactériostatique ou antiseptique, qui peuvent être utiles aussi bien au cours de la conservation que lors de l'insémination dans les voies génitales de la femelle.

Les sulfamides se placent dans cette famille de substances, quoique KNOD'T et SALISBURy (I946) aient montré qu'ils ont une action propre sur le métabolisme des spermatozoïdes de Taureau. L'amélioration de la conservation du sperme de Verrat pur qu'ont obtenu Niwa, MizuHo et IsHIKAWA (1952) avec la Sulfamerazine et l'Homosulfamide, peut être 
interprétée également comme le fruit d'un abaissement du métabolisme du spermatozoïde. Quoiqu'il en soit, avec la dose de $300 \mathrm{mg}$ pour Ioo cc de dilueur (proche de la limite de solubilité) nous n'avons obtenu, avec le dilueur bicarbonate-jaune d'œuf, aucun effet utile ou nuisible du paraminophényl-sulfamide durant les 4 premiers jours de conservation. L'addition de la sulfamide permet une conservation plus régulière des échantillons en évitant les chutes brutales de motilité que l'on constate dans certains tubes généralement à la suite de prolifération bactérienne.

C'est surtout par souci d'antisepsie au cours de l'introduction du sperme " in utero " chez la Truie, que nous avons ajouté de la streptomycine et de la pénicilline aux dilueurs. Aussi est-ce la dose maximum utilisable sans danger pour le sperme que nous avons cherché à déterminer.
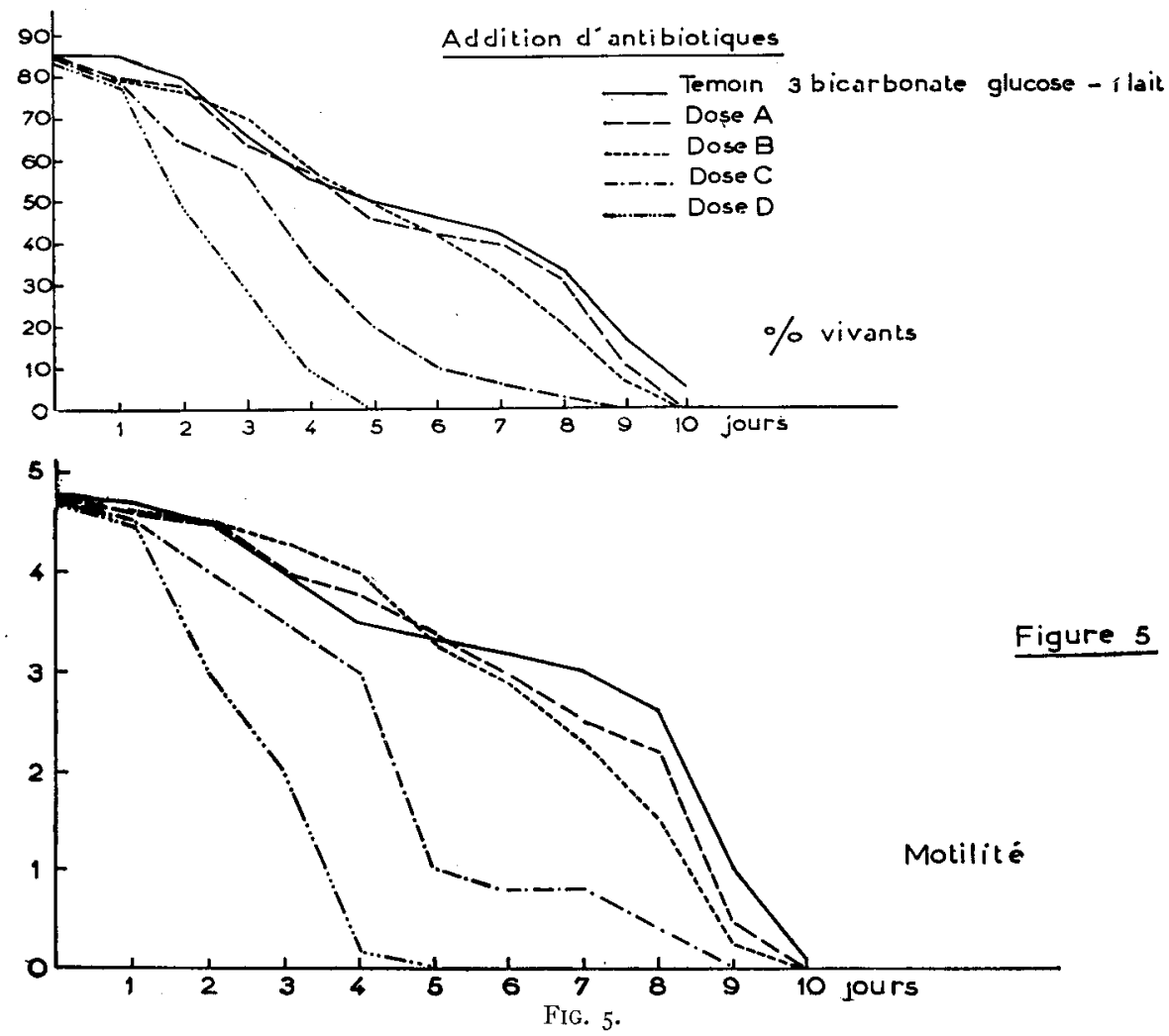

Les quantités employées ont été les suivantes (en $\mathrm{mg} / \mathrm{Ioo}$ cc de dilueur) :

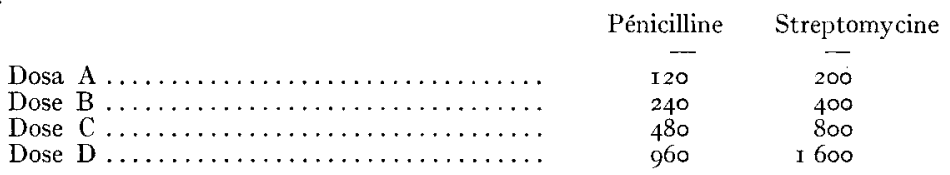

L'examen de la figure 5 montre que les deux plus fortes doses ont un 
effet nuisible très marqué sur la conservation du sperme, effet qui peut être dû en partie à l'hypertonicité du milieu. Les 2 autres doses n'ont pas d'action.

Pour la pratique de l'insémination, nous avons donc employé la dose B (Du MESNIL DU BuIsson et DAUZIER, I955), ce qui conduit à utiliser par Truie, I $g$ de streptomycine et I ooo ooo U. I. de pénicilline.

\section{II. - Conditions de température et de dilution :}

La facilité d'examen et la bonne conservation obtenue dans le milieu 3 bicarbonate-I jaune d'œuf, nous ont poussés à 1'utiliser comme milieu de dilution du sperme pour les essais ultérieurs. La fraction la plus riche en spermatozoïdes, diluée au I/Io a été seule utilisée, $\left(75000 / \mathrm{mm}^{3}\right.$ en moyenne dans les diluats).
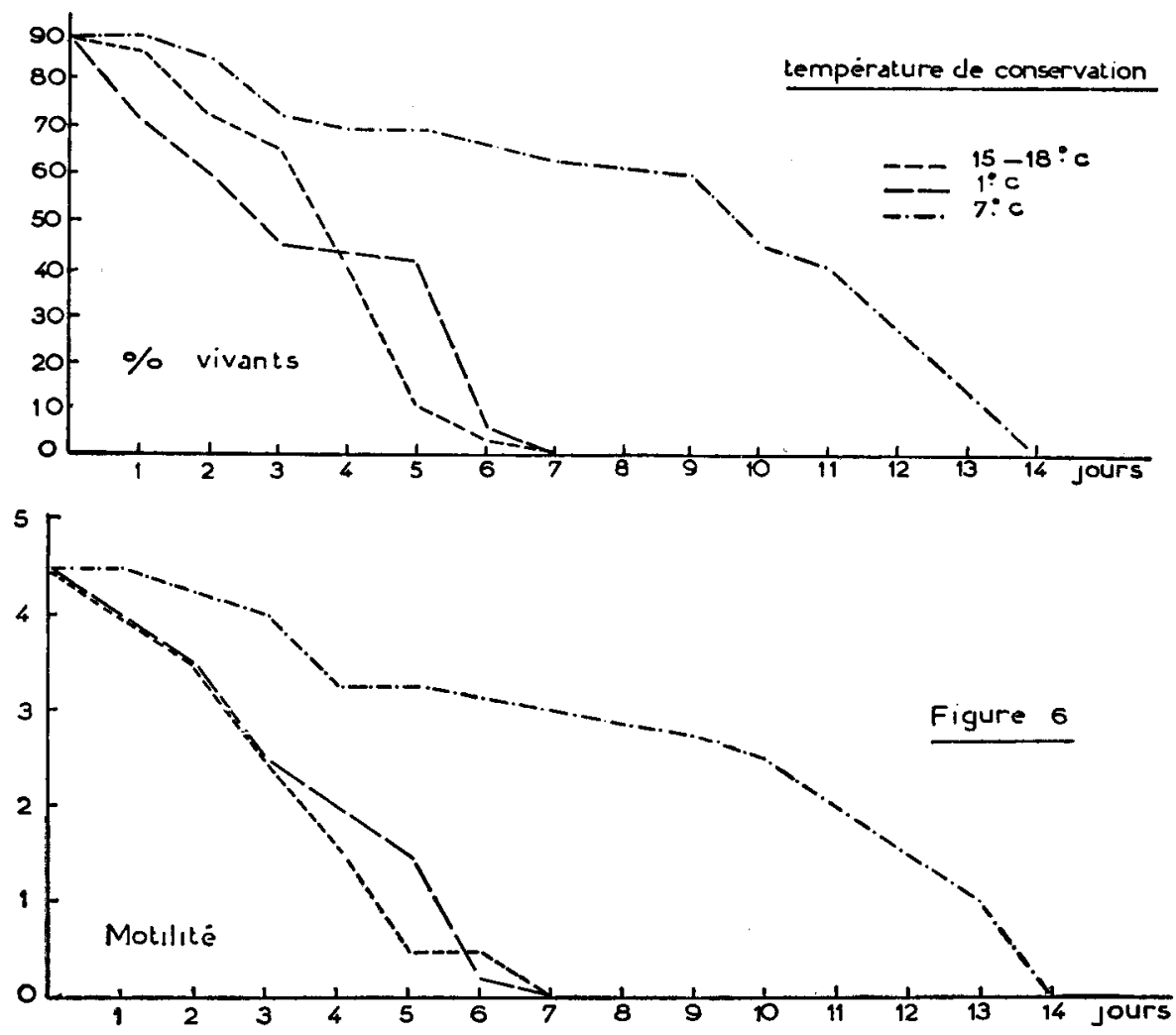

FIG. 6.

Io Température de conservation (fig. 6). - Nous avons fractionné chaque diluat entre 3 parties, conservées aux températures suivantes :

- température ambiante $15-\mathrm{I} 8^{\circ} \mathrm{C}$,

$-\mathrm{I}^{0} \cdot 2^{\circ} \mathrm{C}$,

$-7^{0}-8{ }^{\circ} \mathrm{C}$. 
Les échantillons ont été placés au milieu d'une masse d'eau de 2,5 1 environ a fin d'obtenir un refroidissement progressif, et comparable, d'un échantillon à l'autre.

A la température de I à $2^{\circ} \mathrm{C}$, la conservation est mauvaise. La motilité comme la survie s'abaissent régulièrement depuis le moment de la dilution, et le $7^{\mathrm{e}}$ jour, le sperme est mort.

Les échantillons conservés à $7^{\circ} \mathrm{C}$ se montrent supérieurs aux autres d'un bout à 1'autre de la période de conservation. Très bons durant les 3 premiers jours, ils perdent une partie de leur motilité pour se maintenir

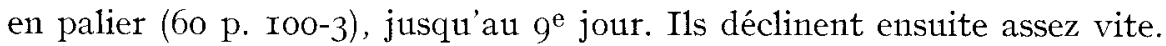

Enfin, à la température ambiante, l'analyse au cours de la conservation est impossible, à cause du développement bactérien. I e premier jour, cependant, le pourcentage de vivants reste voisin de celui du sperme conservé à $7^{\circ} \mathrm{C}$. Ensuite, une grande partie des tubes est polluée par la flore microbienne, la conservation devient mauvaise et dès le $5^{\mathrm{e}}$ jour, il ne reste plus que Io $\mathrm{p}$. Ioo de spermatozoïdes à peine motiles.

Ces expériences confirment la fragilité du sperme de Verrat, aux basses températures, caractéristique déjà signalée par McKENzIE, Milieir et Bauguess (r938) et Ito, Niwa et Kudo (I949). Pour le sperme total, non dilué, ces auteurs recommandent des températures de conservation de $\mathrm{I} 2^{\circ} \mathrm{C}$ et $\mathrm{I}^{\circ} \mathrm{C}$. De nos expériences, il ressort que les échantillons dilués avec des milieux à base de jaune d'œuf ou de lait, peuvent supporter une température nettement inférieure. De son côté, POLGE indique qu'une température de $5^{\circ} \mathrm{C}$, bien qu'extrêmement nuisible à la conservation du sperme entier non dilué, peut être employée pour le sperme dilué avec du jaune d'œuf ou pour la partie la plus riche en spermatozoïdes de l'éjaculat, même conservée pur.

$2^{\circ}$ Mode de refroidissement. - Nous avons amené le sperme à la température de $8^{\circ} \mathrm{C}$ de trois manières différentes : de $30^{\circ}$ à $I 5^{\circ} \mathrm{C}$ en $I / 2$ heure, en I heure $I / 2$ ou en 3 heures $I / 2$ (Vitesses de refroidissement $A, B, C)$.

Le refroidissement le moins rapide (C) se montre (fig. 7) le meilleur pour la conservation ultérieure du sperme. En ralentissant encore la vitesse de refroidissement, durant les 2 premières heures, la capacité de conservation du sperme ne se trouve cependant pas augmentée. Au contraire, après le refroidissement le plus brutal $(\mathrm{A})$, le pourcentage de vivants est considérablement diminué dès le lendemain de la collecte. Quoique la motilité soit également plus faible, on constate alors le même genre de palier entre le $2^{\mathrm{e}}$ et le $5^{\mathrm{e}}$ jour que pour le lot $\mathrm{C}$ : le choc de température a tué une partie des spermatozoïdes, les autres se conservent à peu près normalement. En présence de lait écrémé, avec le refroidissement rapide, les spermatozoïdes sont morts dès le $4^{\mathrm{e}}$ jour de conservation, alors 
que dans les conditions $\mathrm{C}$, on retrouve des spermatozoïdes vivants au bout de ro jours.

Ce dernier résultat confirme ceux de LASLEY et BOGART (I944) qui signalent pour les spermatozoïdes de Verrat, 1'action protectrice du jaune d'œuf contre un choc de température (Io minutes à $I^{\circ} \mathrm{C}$ ) : dans leur expérience, le pourcentage de vivants dans le sperme brutalement refroidi est de 32 p. Ioo et $13 \mathrm{p}$. Ioo avec et sans jaune d'cuf.
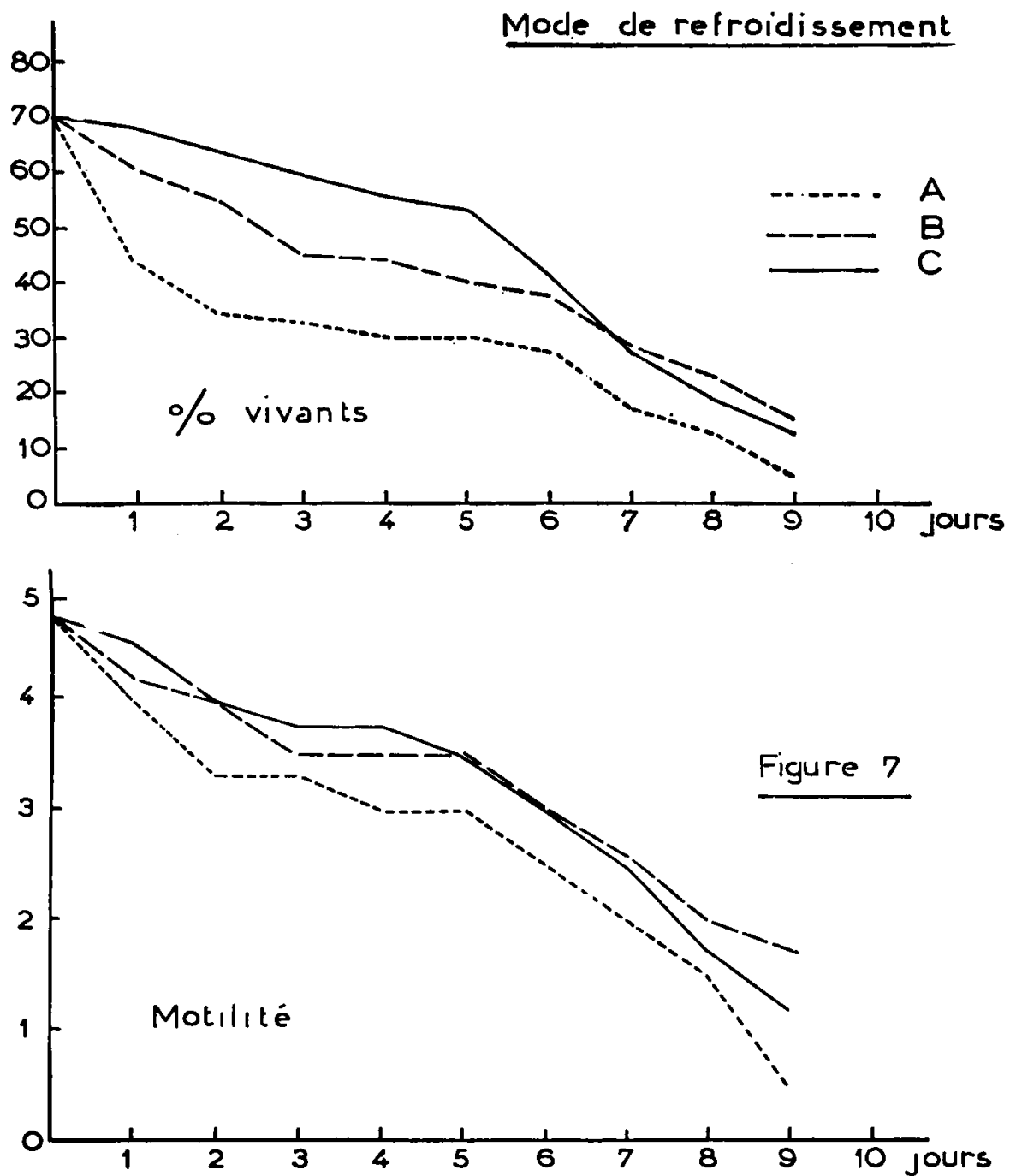

FIG. 7 .

$3^{\circ}$ Taux de dilution. - Nos essais de dilution (fig. 8) ont été réalisés à la température de $7^{\circ} \mathrm{C}$ après refroidissement optimum. 
L'examen des courbes permet de tirer plusieurs conclusions :

Un taux de dilution très faible ( $I$ volume de dilueur pour I volume de sperme) entraîne très rapidement la mort apparente d'une grande partie des spermatozoïdes; dès le surlendemain de la collecte, il ne reste plus que $30 \mathrm{p}$. Ioo des spermatozoïdes motiles.

Il y a peu de différence de conservation entre le sperme dilué au $\mathrm{x} / 5$ et au I/Io. Le sperme amené à Io fois son volume accuse cependant un léger avantage, principalement pour le pourcentage de vivants.
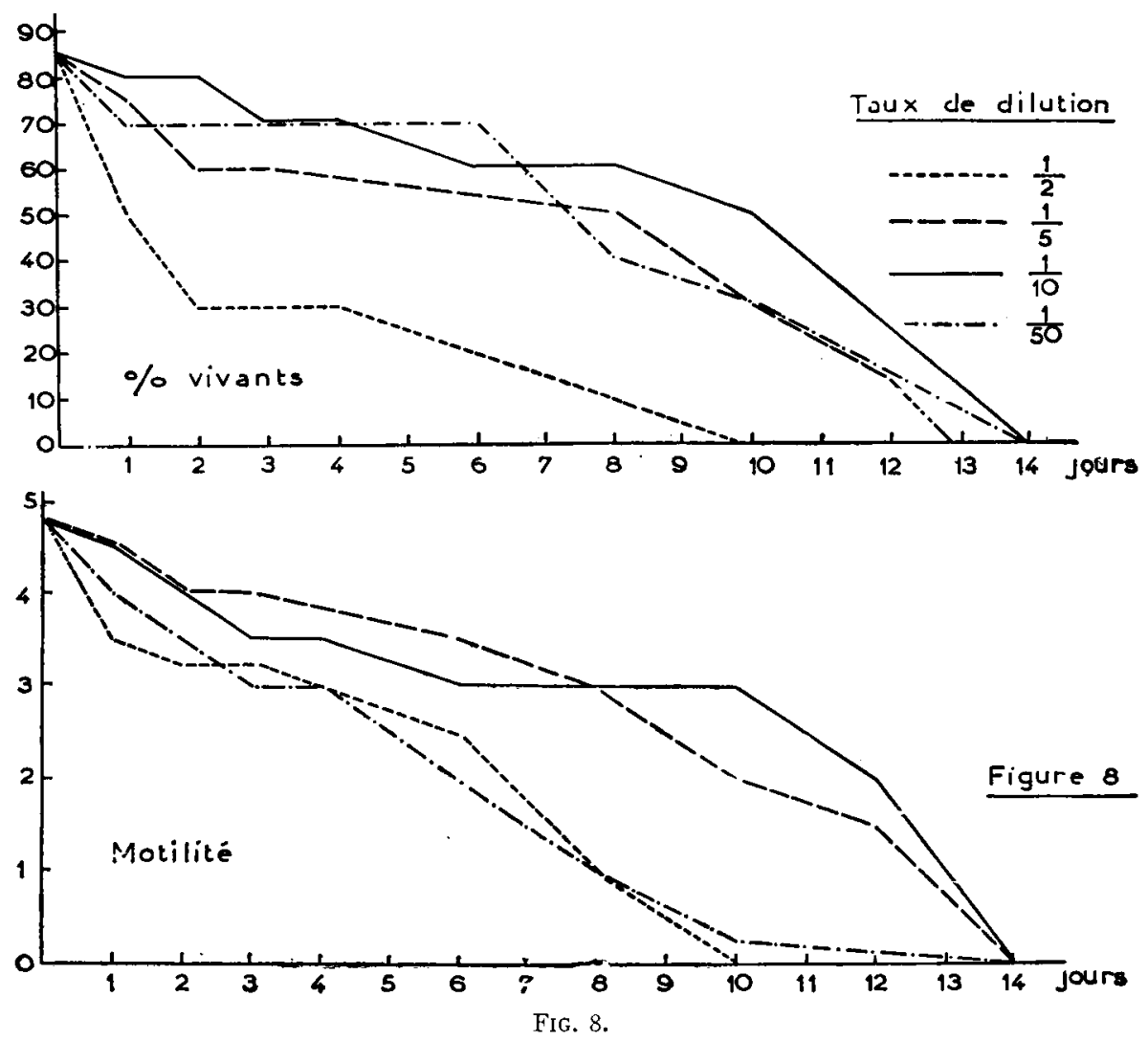

I a dilution au $\mathrm{I} / 5^{\circ}$ provoque un abaissement très net de la motilité alors que le pourcentage de vivants reste comparable à celui que l'on trouve avec les 2 dilueurs précédents. Il est possible que dès ce taux de dilution l'effet propre de la dilution (EMmEns et SwYER, 1948) se fasse sentir malgré la présence du glucose et du jaune d'œuf. Une étude de l'effet de dilution dans d'autres milieux serait nécessaire pour connaître la réaction du sperme de Verrat aux fortes dilutions, car s'il réagit comme le sperme de Taureau, on peut s'attendre à des effets très différents, suivant les ions présents dans le milieu (BISHOP, 1954). 
De ces observations sur la composition du dilueur et les conditions de milieu favorables à la survie du sperme, on pouvait tirer une technique de conservation des spermatozoïdes " in vitro ". Une question restait posée, la plus importante, pour l'utilisation pratique : le pouvoir fécondant est-il préservé en même temps que la motilité ?

\section{III. - Maintien du pouvoir fécondant :}

Les essais d'insémination tentés par ITo, NIwA et Kudo (I949) laissaient présager une réponse affirmative. En effet, ces auteurs ont obtenu 34, 7 p. roo de fécondation avec du sperme conservé pur pendant 3 jours. Or, à ce moment, la survie des spermatozoïdes et la motilité de la majorité, semblent extrêmement faibles ; l'agitation prolongée à une température voisine de celle du corps permet, il est vrai, de les ranimer et explique ces résultats. Avec le sperme conservé dilué, en présence de lait et de jaune d'œuf, la vitalité des spermatozoïdes reste bonne pendant au moins 3 jours, et l'examen immédiat est suffisant pour le constater. On pouvait craindre cependant qu'il n'existe que peu de corrélation entre pouvoir fécondant et motilité du sperme conservé, comme c'est le cas pour le sperme de Lapin (EL SHEIK et CASIDA, I954) ou celui de Bélier (Dauzier, Thibaul'T et Wintemberger, I954).

Seule, l'insémination systématique de Truies avec du sperme conservé dilué pouvait permettre de tester le maintien du pouvoir fécondant. Nous l'avons pratiqué d'une part sur des Truies d'élevage conservées jusqu'à la mise-bas, d'autre part sur des Truies de Ioo $\mathrm{kg}$, abattues au bout de 3 jours, pubères en cestrus, ou impubères après traitement gonadotrope (DU MESNIL DU BuIsson, I954). La résorption des dilueurs dans le tractus génital des truies semble se faire facilement dans les 2 cas.

Avec 3 des dilueurs précédemment cités, additionnés de pénicilline et de streptomycine, à la dose indiquée plus haut, après conservation maximum de 6 heures, nous avons obtenu avec DAUZIER, les pourcentages suivants de mise-bas sur I 45 Truies:

3 Bicarbonate-glucose-I lait : 37,8 p. Ioo,

I Glycocolle-I lait : 48,8 p. Ioo,

2 Glycocolle-I jaune d'œuf : 53,6 p. Ioo, le nombre moyen de porcelets par portée étant de $9, \mathbf{I}$.

Pour ce dernier dilueur, nos résultats sont voisins de ceux de PoLGE (I956) : sur II 8 inséminations avec ou sans antibiotiques, 49, I p. Ioo ont été suivies de gestations.

Ces chiffres permettent d'apprécier l'efficacité globale de la méthode d'insémination plus que le pouvoir fécondant du sperme, car la résorption fœtale précoce ou tardive a déjà joué. Ces pourcentages constituent néanmoins des minima de fécondation. 
Pour apprécier plus directement le pouvoir fécondant du sperme, nous avons abattu un certain nombre d'animaux trois jours après l'insémination.

Une première série de Truies a été inséminée avec du sperme conservé 2 à 5 heures, d'une part dans le 3 bicarbonate-glucose-I lait (I5 animaux), d'autre part dans le 3 bicarbonate-glucose-I jaune d'œuf ( 8 animaux). Chez 2I Truies sur 23, on a retrouvé des œufs fécondés : 69,8 p. Ioo des oufs étaient segmentés avec le premier dilueur et 60,8 p. roo avec le second. Ces truies étaient en ostrus au moment de l'insémination.

Ensuite, nous avons tenté une expérience sur le matériel très particulier que constitue la Truie pré-pubère, traitée avec des hormones gonadotropes. Nous avons dû, à l'abattage, éliminer toutes les Truies

TABLEAU II

Maintien du pouvoir fécondant

\begin{tabular}{|c|c|c|c|c|c|c|c|}
\hline \multirow[b]{2}{*}{ Dilueurs } & \multirow[b]{2}{*}{$\begin{array}{l}\text { Etat des } \\
\text { truies }\end{array}$} & \multirow[b]{2}{*}{$\begin{array}{l}\text { Durée de } \\
\text { conser- } \\
\text { vation }\end{array}$} & \multicolumn{2}{|c|}{ Truies } & \multicolumn{3}{|c|}{ (Eufs } \\
\hline & & & Inséminées & $\begin{array}{l}\text { Ayant des } \\
\text { œufs } \\
\text { fécondés }\end{array}$ & Retrouvés & Fécondés & $\begin{array}{l}\% \text { d'oufs } \\
\text { fécondés } \\
\text { œufs } \\
\text { retrouvés }\end{array}$ \\
\hline $\begin{array}{c}\text { Bicarbonate- } \\
\text { Glucose } \\
\text { I } / 4 \text { œufs }\end{array}$ & \multirow[t]{2}{*}{$\underset{\text { eestrus }}{\text { En }}$} & $2-5 \mathrm{~h}$ & 8 & 8 & 102 & 62 & $60,8 \%$ \\
\hline \multirow{4}{*}{$\begin{array}{l}\text { Bicarbonate- } \\
\text { Glucose } \\
\text { I/4 lait }\end{array}$} & & $2 \cdot 5 \mathrm{~h}$ & 15 & $\mathrm{I} 3$ & 169 & I 18 & $69,8 \%$ \\
\hline & \multirow{4}{*}{$\begin{array}{c}\text { Prépubères, } \\
\text { traitées } \\
\text { avec des } \\
\text { hormones } \\
\text { gonado- } \\
\text { tropes }\end{array}$} & $3 \mathrm{~h}$ & 8 & 8 & 69 & $5^{\mathrm{I}}$ & $73,9 \%$ \\
\hline & & $24 \mathrm{~h}$ & 6 & 5 & $5^{1}$ & 30 & $58,8 \%$ \\
\hline & & $48 \mathrm{~h}$ & 6 & 5 & 48 & $3^{\circ}$ & $62,5 \%$ \\
\hline $\begin{array}{c}\mathrm{NaCl} \\
\left(8,5^{\%} \%\right) \\
\text { au moment } \\
\text { de l'emploi }\end{array}$ & & $\mathrm{I}-7 \mathrm{~h}$ & 30 & 24 & 210 & 118 & $5^{6,2} \%$ \\
\hline
\end{tabular}

qui n'avaient pas répondu d'une manière normale au traitement hypophysaire et présentaient des ovaires ou un endothélium utérin très peu différenciés, ou des ovules encore retenus dans un bouchon muqueux, semblable au "gelatin-like plug " décrit par Tanabe, Warnick, Casida et Grummer (1949). 50 femelles ont réagi de façon normale. Les unes (30) ont été inséminées avec du sperme conservé pur, pendant I à 3 heures et dilué avec $\mathrm{NaCl}$ à 8,5 p. I ooo au moment de l'insémination: elles nous ont servi de témoins. Les autres ont été inséminées avec du sperme conservé à $8^{\circ} \mathrm{C}$, dilué dans le 3 bicarbonate-glucose-r lait, pendant 3 heures, 24 heures et 48 heures. Les résultats sont portés sur le tableau II. 
Le nombre d'animaux de chaque lot (6 et 8 ) est trop faible pour tirer une conclusion relative au pourcentage de fécondité à attendre de la semence, en fonction de la durée de la conservation. On voit cependant que la presque totalité (5 sur 6 et 8 sur 8 ) des animaux ont des œufs fécondés trois jours après l'insémination. La proportion des œufs fécondés par rapport aux œufs retrouvés est, dans les différents lots, du même ordre que chez les témoins (56 p. Ioo). On peut affirmer que le pouvoir fécondant du sperme de Verrat subsiste au bout de 48 heures de conservation dans le dilueur au lait.

En utilisant du sperme conservé non dilué ITo, Niwa et Kudo (I949) ont montré que dans ces conditions également le pouvoir fécondant du sperme de Verrat est susceptible de se maintenir parfaitement au moins 48 heures $(62,5$ p. Ioo de Truies pleines après insémination avec $\mathrm{du}$ sperme conservé 24 à 48 heures).

Inversement, POLGE (I956) enregistre un abaissement notable de succès des inséminations faites avec du sperme conservé seulement 24 heures à 30 heures ( 29 p. IOo) dans un dilueur à base de jaune d'œuf, et n'obtient qu'exceptionnellement des gestations (3 sur 28) après 48 heures de conservation du sperme. Il semble que la présence de lait assure une meilleure conservation du pouvoir fécondant que celle du jaune d'œuf, comme c'est le cas pour le sperme de Bélier (DAUzIER, I956).

Ainsi, il est possible que dans la pratique de l'insémination pour une durée de conservation de 24 à 48 heures, on soit amené à écarter certains dilueurs peut-être nuisibles à la conservation du pouvoir fécondant ou même à conserver la semence non diluée. Le facteur dilution n'en reste pas moins un élément d'économie indispensable à la mise en pratique de l'insémination artificielle porcine.

Malheureusement, le fait qu'un sperme permette une fécondation régulière des cufs n'entraîne pas forcément une production normale à la mise-bas. D'autres problèmes se posent, encore entiers ou en partie résolus, entre autres celui de la détection des chaleurs en dehors du Verrat, et celui du maintien de la gestation en relation avec la résorption fotale précoce.

\section{RÉSUMÉ}

En résumé on obtient une bonne conservation de la motilité des spermatozoïdes du Verrat dans les conditions suivantes :

- Refroidissement des échantillons au sein d'une masse de 2 à 3 litres d'eau dont la température initiale est aux environs de $3 \mathrm{I}^{\circ}$ et que $1^{\prime}$ on place à $7^{\circ} \mathrm{C}$.

- Température de conservation : $7^{\circ} \mathrm{C}$.

- Taux de dilution compris entre $\mathrm{I} / 5$ et $\mathrm{I} / \mathrm{IO}$. 
Dans les conditions d'examen où nous nous sommes placés, 1'apport d'un milieu biologique dans le dilueur semble nécessaire pour permettre aux spermatozoïdes de retrouver leur motilité après conservation prolongée.

L'adjonction de substances existant dans le sperme (ester glycérophosphorique de la choline, inositol) ou celle d'antibiotiques chimiques ou biologiques n'améliore pas la conservation.

Les dilueurs étudiés, à base de lait ou de jaune d'œuf, contenant du glucose et du bicarbonate, ou du glycocolle, permettent à la fois la conservation prolongée de la motilité ( $\mathrm{x}$ à 2 semaines) et le maintien dans de bonnes conditions du pouvoir fécondant pendant au moins 5 à 6 heures.

Bien que nous ayons obtenu des fécondations après 48 heures de conservation, des expériences sur un plus grand nombre d'animaux seront nécessaires pour confirmer le rôle du lait dans le maintien du pouvoir fécondant.

Reçu pour publication le 22 mai $\mathbf{1 9 5 6 .}$

\section{RÉFERENCES BIBLIOGRAPHIQUES}

Bishop (M. W. H.), I954. - Studies on fertility, 6, 8I-95.

DAuzIER (L.), I956. - III Cong. Inter. Reprod. Anim. (sous presse).

Dauzier (L.), Thibault (C.) et Wintemberger (S.), 1954. - Ann. Endocrin., 15, 34I-350.

Diament (M.), I954. - Thèse Fac. Sci. Paris, I vol.

DU Mesnil DU Buisson (F.), I954. - Ann. Endocrin., 15, 333-340.

Du Mesnil du Buisson (F.), Dauzier (L.), I955. - C. R. Acad. Sci., 241, I $867-1869$.

El-Sheikh (A. S.) et Casida (I. E.), I954. - J. Anim. Sci., 13, 660-667. Emmens (C. W.) et Swyer (G. I. M.), I948. - J. Gen. Physiol. U. S. A., 32, I2I-I 38 .

ITo (S.), Niwa (T.) et Kudo (A.), I949. - Jap. J. Zootech. Sci., 19, II3-Ir8. Kahn (E.) et Lévy (J.), I936. - C. R. Acad. Sci., 202, 2186-2187.

Kampschmid' (R. F.), Mayer (D. T.), Herman (H. A.) et Dickerson (G. E.), I95I. - J. Dairy Sci., 34, 45-5I.

Knodt (C. B.) et Salisbury (G. W.), I946. - J. Dairy Sci., 29, 285-29I.

LASLEY (J. F.) et Philims (P. H.), I939. - Missouri Exp. Sta. J., 567.

Lasley (J. F.) et Bogart (R.), I944. - Am. J. Physiol., 141, 6I9-624.

MANN ('T.), I95I. - Nature, 168, I043-I044.

MANN (T.) et LEONE (E.) I953. - Bioch. J., 53, I40-I48.

McKenzie (F. F.), Milleer (J. C.) et Bauguess (L. C.), I938. - Un. Missouri Agric. Exp. Sci. Res. Bull., 279.

Milovanov (V. K.), I932. - Prob. Zhivot,, 4, 3 I (A. B. A., 1, II2).

Milovanov (V. K.), I933. - "Artif. Insemin. in Livestock ", Moscou.

Niwa (T.), Mizuho (A.) et Ishikawa (S.), I952. - Bull. Nat. Inst. Agric. Sci., Chiba Japan, 3, Ioo-Io7.

Nol. (D. O.), I949. - Phillip. J. Anim. Ind., 10, 247-259.

Nozaki (H.), Kawase (T.), Nrwa (T.), I952. - Bull. Nat. Inst. Agric. Sci. Chiba Japan, 3, Io8-IIg. 
Philitips (P. H.) et Lardy (H. A.), r940. - J. Dairy Sci., 23t 399-404.

Polge (C.), I956. - Vet. Rec., 68, I-I5.

Rodolfo (A.), I934. — Phillip. J. Sci., 53, I83-203.

Roy (A.), I955. - Vet. Rec., 67, 330-331.

Salisbury (G. W.), Fuller (H. K.) et WilletT (E. L.), I94I. - J. Dairy Sci., 24, 905-9I0.

Sekine (T.), I95I. - J. Biochem. (Tokyo), 38, I7I-I79.

Tanabe (T. Y.), Warnick (A. C.), Casida (L. E.), Grummer (R. H.), I949. - J. Anim. Sci., 8, 550-557.

Thibault (C.), i955. - "Exposé actuel de Biologie cellulaire ". Masson, Paris, 306-334. 